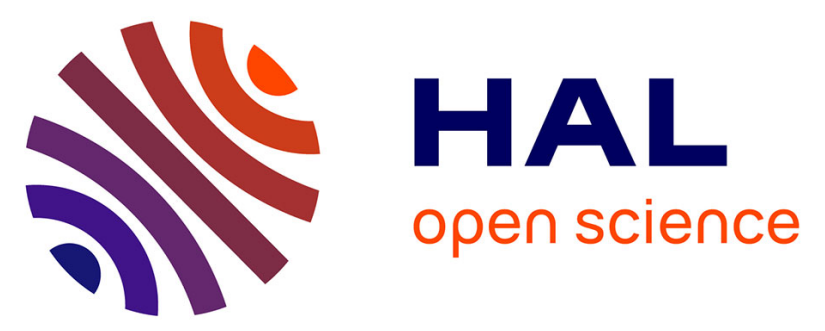

\title{
L'éducation à la santé familiale, modélisation et expérimentation de nouvelles interventions éducatives à porter soins et secours L'éducation à la santé familiale auprès d'adolescents
}

Sebastien Riquet, Nicolas Brun, François Frété, Christine Ammirati, Rémi

Gagnayre, Jean-François d'Ivernois

\section{To cite this version:}

Sebastien Riquet, Nicolas Brun, François Frété, Christine Ammirati, Rémi Gagnayre, et al.. L'éducation à la santé familiale, modélisation et expérimentation de nouvelles interventions éducatives à porter soins et secours L'éducation à la santé familiale auprès d'adolescents. Éducation thérapeutique du patient / Therapeutic patient education , 2016, 8 (2), pp.20107. 10.1051/tpe/2016014 . hal-01799698

\section{HAL Id: hal-01799698 \\ https://sorbonne-paris-nord.hal.science/hal-01799698}

Submitted on 25 May 2018

HAL is a multi-disciplinary open access archive for the deposit and dissemination of scientific research documents, whether they are published or not. The documents may come from teaching and research institutions in France or abroad, or from public or private research centers.
L'archive ouverte pluridisciplinaire HAL, est destinée au dépôt et à la diffusion de documents scientifiques de niveau recherche, publiés ou non, émanant des établissements d'enseignement et de recherche français ou étrangers, des laboratoires publics ou privés. 


\title{
Article original/Original article
}

\section{L'éducation à la santé familiale, modélisation et expérimentation de nouvelles interventions éducatives à porter soins et secours}

\section{L'éducation à la santé familiale auprès d'adolescents}

\author{
Sébastien Riquet ${ }^{1,2 \star}$, Nicolas Brun ${ }^{3}$, François Frete ${ }^{4}$, Christine Ammirati ${ }^{1,5}$, Rémi Gagnayre ${ }^{1}$, \\ Jean-François d'Ivernois ${ }^{1}$ \\ 1 Université Paris 13, Sorbonne Paris Cité, Laboratoire Éducations et Pratiques de Santé EA 3412, 93017 Bobigny, France \\ 2 Aix-Marseille Université, École universitaire de maïeutique Marseille Méditerranée, 12384 Marseille, France \\ 3 Union Nationale des Associations Familiales, 75009 Paris, France \\ 4 Caisse Centrale de la Mutualité Sociale Agricole, 93547 Bagnolet, France \\ 5 SimUSanté, CHU Amiens-Picardie, Université de Picardie, 80054 Amiens, France
}

(Reçu le 27 juin 2016, accepté le 7 novembre 2016)

\begin{abstract}
Résumé - Introduction : Le Laboratoire Éducation et Pratiques de Santé (LEPS, EA 3412), associé à l'Union Nationale des Associations Familiales (UNAF) et à la Mutualité Sociale Agricole (MSA) propose un nouveau modèle d'Éducation à la Santé Familiale (ESF), fondé sur des compétences répondant au besoin de réappropriation par les familles de savoir-faire en soins et secours de proximité. Plusieurs réunions d'experts ont abouti à la modélisation d'interventions éducatives, comptant entre 9 à 12 heures de formations, réparties entre 4 modules obligatoires et 3 optionnels. Un module est spécifiquement destiné aux jeunes, leur permettant d'agir comme un acteur santé au sein de leur famille. Méthode : Ce modèle a été expérimenté auprès de 21 lycéens, d'un établissement éducatif dépendant de l'Union Nationale des Maisons Familiales et Rurales (MFR). Résultats : À l'issue de cette formation ESF, on a constaté que les adolescents se sentent davantage aptes et confiants à porter soin et secours. Le questionnaire d'enquête a montré une augmentation de leur sentiment de compétence vis-à-vis de la gestion d'une situation d'urgence et des problèmes de santé courants. Ce sentiment de compétence, interrogé 3 mois plus tard par entretiens téléphoniques a été confirmé. Il a pu être établi une liste des principales situations, pour lesquelles les adolescents se sont portés soins et secours, tout en réévaluant leur sentiment d'efficacité personnelle. Conclusion : Les résultats de cette enquête témoignent de l'atteinte des objectifs de l'ESF. La validité du concept d'ESF, auprès d'autres groupes d'adolescents, devra continuer d'être explorée.
\end{abstract}

Mots clés : éducation à la santé familiale / adolescents / compétences en santé

\begin{abstract}
Family Health Education, modeling and experimentation with new educational interventions to bear care and aid Family health education with teenagers. Introduction: The Education and Health Practice Laboratory (LEPS, EA 3412), together with the National Union of Family Associations (UNAF) and the Social and Agricultural Insurance (MSA) offers a new model of Family Health Education (ESF) based on skills responding to the need of re-appropriation by the families of care and emergency competencies. Several expert's meetings led to the modeling of educational interventions with 9-12 hours of training spread among 4 compulsory and 3 optional modules. A module was specifically designed for teenagers, enabling them to act as health actor in their family. Method: This model was tested on 21 students of an educational institution that depends on the National Union of Rural Families Homes (MFR). Results: At the end of the ESF we found that teenagers feel more able to bring care and relief. The survey showed an increase in their feeling of being competent in managing the emergencies and the common health problems. This feeling of compétence was confirmed three months later by telephone conversations.
\end{abstract}

^ Correspondance : Sébastien Riquet, sebastienriquet@yahoo.fr 
A list of the main situations in which teenagers have brought care and relief and reassessed their self-efficacy was made. Conclusion: The results of this survey demonstrate the achievement of the ESF objectives. The validity of the concept of ESF for other groups of teenagers should continue to be explored.

Key words: family health education / teenagers / health competences

\section{Introduction}

«Depuis toujours, les adultes apprennent aux enfants à se nourrir, se soigner, se défendre, se bien comporter avec leurs semblables... » [1].

Ces pratiques, essentielles à la survie des groupes humains, se retrouvent dans toutes les cultures. Depuis l'antiquité, la transmission familiale ou domestique est donc constituée des processus éducatifs au sein de la famille pour préparer les individus à leur vie quotidienne et en communauté. Dans ce qui constitue «l'éducation familiale», les pratiques domestiques de santé y jouent un rôle fondamental [2]. Depuis 2006, le Laboratoire Éducations et Pratiques de Santé (LEPS, EA 3412) travaille sur un nouveau modèle dans le champ des éducations en santé [3], classiquement constitué de trois activités : 1'éducation à la santé [4], l'éducation thérapeutique du patient [5] et l'éducation à porter secours (secourisme) [6].

L'éducation «à porter soins et secours » (EPSS, appelée ultérieurement : Éducation à la Santé Familiale (ESF)) constitue donc un nouveau type d'intervention éducative, dont le but est une réappropriation par les familles et les adolescents de compétences en santé au regard du contexte socio sanitaire actuel. Elle est décrite ainsi par d'Ivernois et Gagnayre [7] :

«L'EPSS concerne les domaines de la prévention, des soins et du secourisme élargi appliqués à l'espace familial et de proximité de vie. Elle a un caractère opérationnel et d'application immédiate. Elle est dirigée vers l'autre, fondée sur la notion qu'au-delà du maintien de sa propre santé, il est essentiel d'apporter la santé à autrui (en particulier aux bébés, aux enfants et aux personnes âgées). En cela, elle constitue une éducation en santé familiale. L'EPSS forme à une vigilance applicable aux soins du quotidien comme aux situations d'exception (canicule, grand froid, épidémie); elle prépare à repérer et à faire face aux situations d'urgence, mais aussi aux problèmes de santé courants et aux petits maux de la vie quotidienne. »

L'EPSS correspond donc aux interventions éducatives informelles [8] d'une des plus anciennes éducations : l'éducation à la santé familiale (ESF). L'appellation ESF est venue se substituer à celle d'EPSS dans le cadre d'une démarche de modélisation [9].

Pour constituer le modèle, une première enquête quantitative [7] de portée nationale effectuée par le LEPS en association avec l'Union Nationale des Associations Familiales (UNAF) et la Mutualité Sociale Agricole (MSA) a démontré à 72,4\% le souhait et les besoins de 669 familles à bénéficier d'une ESF. Une seconde enquête [10] utilisant la même méthodologie dans un territoire rural a rapporté les mêmes conclusions. Celle-ci a montré, de plus, l'intérêt (88\%) des adoles- cents $(n=103)$ participant aux familles interrogées $(n=169)$ de pouvoir bénéficier d'une ESF.

Enfin, une enquête qualitative a été menée au moyen de 3 focus groupes constitués de panels familiaux : des parents de jeunes enfants $(n=7)$, des parents ayant en charge un ainé $(n=7)$ et des adolescents $(n=7)$. Cette dernière étude [11] a permis d'identifier quatre grands domaines de santé dans lesquels les membres d'une famille s'entraident au quotidien : les urgences, les maux de santé courants, les situations de santé de gravité intermédiaire et les problèmes de nature psychologique.

À partir de l'ensemble de ces résultats et de plusieurs réunions d'experts réunissant médecins urgentistes (3), de santé publique (1), pédagogue de la santé (1), sage-femme (1), infirmières (2) et représentants d'associations familiales (3), il a été possible d'élaborer un programme expérimental de formation modulaire [12] à la santé familiale.

\subsection{Présentation du programme de formation d'éducation à la santé familiale}

Ce programme d'ESF, d'une durée de 9 à 12 heures, est constitué de deux parties [9] :

I. un tronc commun de 4 modules obligatoires :

- «Santé et famille au quotidien » réunissant les maux de santé courants,

- «Traumatismes et agressions extérieures » abordant la pratique des pansements et les techniques de bandages entre autres,

- «Urgences familiales » (convulsions, arrêt cardio respiratoire, etc.) dans le cadre du lieu de vie,

- «Situations d'exceptions» (grands froids, canicule, accident nucléaire, etc.).

Chaque module à une durée de 2 à 3 heures, sauf « situations d'exception » qui consiste en la remise d'une documentation spécifique.

II. 3 modules spécifiques aux choix des participants en fonction de la constitution de leur famille et de leurs besoins d'apprentissage. Ils représentent par module 3 à 4 heures de formation. Il s'agit des modules :

- «Puériculture et pédiatrie » concernant la santé et les soins au nouveau-né et au jeune enfant. Il est adapté aux jeunes parents ou aux adolescents pratiquant le babysitting dans la fratrie ou en dehors de la famille;

- «Gérontologie et personnes âgées » apparaissant pertinent lorsque les familles ont un parent sénior en charge ; 
- «Agir en tant que jeune » spécifiquement dédié aux adolescents leur permettant d'aborder la santé par ce qu'ils peuvent faire pour eux-mêmes et les autres en se positionnant comme un acteur santé au sein de leur famille et avec leur pair.

L'ESF a recours à une pédagogie active et participative $[13,14]$. Les séances prennent appui sur les savoirs et les expériences profanes des familles en les invitant à approfondir leurs connaissances et à développer une démarche de résolution de problèmes de santé et/ou de gestion de crises qui rompent avec les messages traditionnels d'interdits encore trop souvent utilisés en éducation pour la santé [15]. Les conflits cognitifs qui émanent des échanges entre pairs favorisent l'apprentissage des participants. Il ne s'agit donc pas de «cours » mais bien d'un accompagnement pédagogique vers l'acquisition ou le renforcement de compétences. Ils font appel à des tables rondes, des échanges, des jeux de cartes-réponses, des démonstrations, des ateliers de mises en situation, etc. Les participants reçoivent des documents à emporter à la maison et sont aussi orientés vers les ressources officielles disponibles (sites internet utiles, adresses des IREPS).

Les intervenants en ESF sont des professionnels de santé et du champ social, experts du contenu des modules dans lesquels ils interviennent. Il est nécessaire qu'ils disposent d'une expérience préalable en éducation pour la santé, en éducation thérapeutique ou en pédagogie, auprès des adolescents notamment. Ils auront tous reçu en amont une formation de 3 jours leur permettant de s'approprier la philosophie et la pédagogie de l'ESF. Lors de cette formation, ils simulent des séances de formation ESF en prenant appui sur un ensemble d'outils pédagogiques : diaporama interactif, cartes de questions-réponses, questionnaires d'évaluation. Ces outils élaborés par l'IPCEM, le LEPS EA 3412, l'Institut national de prévention et d'éducation pour la santé (Inpes) et la Sécurité Civile leur sont également remis pour distribution aux familles.

\section{Objectifs}

Dans l'étude que nous présentons, notre objectif a consisté à vérifier auprès d'adolescents leur perception du programme ESF qu'ils ont reçu et le bénéfice qu'ils en ont tiré 3 mois après la formation.

\section{Méthodes}

\subsection{Contexte et intervenants}

En février 2013, un médecin généraliste (titulaire d'un master éducation et santé) et un psychologue (titulaire d'un diplôme universitaire en thérapie comportementale et cognitive) travaillant dans un Pôle de prévention et d'éducation du patient (PPEP) ont été formés à la Caisse centrale de la mutualité sociale agricole (CCMSA), pour intervenir dans un programme
ESF destiné à un groupe d'adolescents. Une formation expérimentale de 2 journées a été conduite en avril 2013 en partenariat avec la Maison familiale et rurale (MFR) de Conty, dans la Somme. Cette formation ESF s'est tenue avec une classe de 21 lycéens préparant un BAC professionnel : «Aide à la personne ». Le binôme de formateurs ESF a été assisté par un enseignant référent pédagogique de la classe de lycéens, également référent sauveteur secouriste au travail (SST).

\subsection{Déroulement des modules du tronc commun}

Pour le module : «Santé et famille au quotidien », l'outil dit des 4 petits tours, adapté de la technique du «Philips $6 / 6 »[16,17]$ a permis de faire travailler les lycéens en 4 groupes, chaque groupe travaillant sur une question et différentes situations en lien avec les contenus du module.

Le module «Traumatismes et agressions extérieures » a été abordé par un «brainstorming » $[17,18]$ sur ce que doit comporter une pharmacie à domicile. Un atelier pour réaliser des bandages a été organisé.

Le module «Situations d'exception » est travaillé en 3 groupes. Chacun doit commenter un thème abordé sur une affiche de l'Inpes, donnée par les formateurs, pour émettre des recommandations. Une restitution en binôme sous forme de questions/réponses avec le groupe classe est ensuite faite utilisant la méthode de « l'exposé oral interactif » [19].

Pour le module «Urgences», 4 situations d'urgence ont été identifiées par la classe. En sous-groupes les lycéens doivent proposer des conduites à tenir qui sont ensuite mises en scènes. Le massage cardiaque est pratiqué en atelier à l'aide de mannequins utilisés en formation de secourisme.

\subsection{Déroulement des modules spécifiques}

Pour le module «Puériculture et pédiatrie», un groupe «d'experts », constitué de lycéens se destinant professionnellement à côtoyer ce public a travaillé en dehors de la classe des situations en lien avec le module. Les autres adolescents de la classe ont abordé ces mêmes questions par «élaboration progressive » [20] donnant un effet boule de neige aux éléments de réponses. Le rapporteur de la classe a ensuite présenté les résultats aux experts qui ont apporté leurs réflexions, avec la modération des formateurs.

Le module «Gérontologie et personne âgées » a été traité selon le même principe.

Le module «Agir en tant que jeune » a été proposé aux lycéens au moyen d'un «photo langage ${ }^{\circledR}$ » [21] produit par l'Inpes. Cette intervention a permis l'émergence d'idées pour agir en tant que jeune pour sa santé et celle de sa famille ou de ses amis. 4 groupes ont été amenés à réfléchir à des slogans leur permettant de se positionner comme un référent en santé. Pour finir, deux panels de discussions improvisés [17,22] ont été constitués en lien chacun avec une thématique différente. Chaque adolescent pouvait choisir son groupe; les formateurs sont garants du débat qui s'y produit. 


\section{4 Évaluation}

À la fin de la formation, deux questionnaires ont été distribués aux adolescents. Le premier évaluait leur satisfaction vis à vis de l'expérience ESF et le second, le sentiment de compétence qu'ils estimaient avoir acquis immédiatement à la fin de la formation.

Puis, à 3 mois de distance, un entretien téléphonique individuel d'une dizaine de minutes a permis d'explorer leur sentiment de compétence vis-à-vis des situations de santé qu'ils ont rencontrées, suite à la formation.

La participation des adolescents à cette étude a fait l'objet d'un consentement éclairé de leur part et celle de leurs parents sur les buts et les modalités des interventions éducatives dispensées.

\section{Résultats}

\subsection{Profil des participants}

Une classe de 21 lycéens a participé aux ateliers ESF. Elle était constituée de 20 filles et d' 1 garçon avec une moyenne d'âge de 17,5 ans.

\section{2 Évaluation de la formation ESF reçue}

Abordée au moyen de 14 items, l'évaluation de la formation s'est révélée très satisfaisante, variant de 52,4 à 95,3\% par l'ensemble des 21 adolescents ( $c f$. Tab. I). Seul le dernier item proposant l'ESF comme une option possible au Baccalauréat n'a pas obtenu une réponse positive par la majorité des adolescents.

\section{3 Évaluation du sentiment de compétence acquis suite à une ESF}

À la fin du programme d'ESF, les adolescents se sentent majoritairement compétents (de $50 \%$ à $100 \%$ ) pour agir sur l'ensemble des situations de santé abordées durant les interventions éducatives (Tab. II). Prendre en charge une luxation (foulure) est la seule situation pour laquelle une majorité d'adolescents ne se sent pas compétent. Par contre, mettre une personne en position latérale de sécurité (PLS) est un geste qui semble maitrisé par tous.

\section{4 Évaluation à trois mois après la formation}

Trois mois après la formation, les 21 adolescents ont été interrogés sur leur téléphone portable. L'entretien téléphonique a porté sur la totalité des items des questionnaires qui leur avaient été proposés immédiatement après la formation. Seuls, 13 adolescents ont répondu à cet entretien. Ils demeurent majoritairement satisfaits (de 53,85 à 84,61\%) de la formation qu'ils ont reçue ( $c f$. Tab. III). La formation en ESF répond encore globalement $(61,54 \%)$ à leurs attentes.

En fonction des problèmes de santé rencontrés sur la période post-formation, les adolescents ont indiqué les situations vis-à-vis desquelles lesquelles ils se sentaient plus ou moins capables d'agir (Tab. IV). Le massage cardiaque, est cité à de nombreuses reprises comme la situation la plus difficile à maitriser. Par contre, mettre une personne en PLS est cité plusieurs fois comme une action «maîtrisée ».

La majorité des adolescents (10/13) interrogés ont rapporté des actions en lien avec le changement de la pharmacie de leur domicile, suite à l'ESF. Ils expliquent, par exemple, avoir amélioré la trousse de secours en y rajoutant du désinfectant, avoir éliminé les médicaments périmés en les ramenant à la pharmacie après avoir vérifié les dates de péremptions, avoir aussi pensé à ne pas laisser les médicaments à portée d'enfants plus jeunes. Seuls, trois d'entre eux n'ont pas modifié la pharmacie du domicile parental suite la formation aux motifs suivants : ne pas avoir eu le temps de le faire ou parce que ce sont leurs parents qui gèrent la pharmacie à la maison.

\section{Discussion}

\subsection{Les limites de l'étude}

Les adolescents ayant participé à ces interventions éducatives en santé présentent un biais de sélection en raison de leur parcours scolaire qui est un Baccalauréat professionnel «Aide à la personne ». Plusieurs d'entre eux ont constaté une répétition entre certains contenus des modules ESF et leur programme de formation : "c'est des trucs qu'on connaissait avant, ils ont pas vraiment développé»; "on avait vu déjà pas mal de choses avant cette formation ». Ainsi, il pourrait être plus pertinent d'offrir en priorité le programme ESF aux adolescents inscrits dans des filières générales des programmes scolaires, car moins enseignés dans le domaine de la santé.

Par ailleurs, la durée post-formation a été très brève et surtout s'est étendue sur la période de mai à juillet au cours de laquelle, comme le montrent les observations de l'InVS [23], on constate une baisse de fréquentation des urgences et des consultations en médecine générale pour des maladies infectieuses telles que la bronchite, les syndromes grippaux, les malaises, les fièvres et certaines allergies. Ce fait se retrouve dans les réponses des adolescents : "non j'ai pas été particulièrement malade, juste un rhume».

\subsection{Contexte scolaire et interventions d'éducation à la santé familiale}

L'ESF à destination des adolescents s'inscrit dans la problématique générale de l'enseignement de la santé dans les programmes scolaires. On note que parmi les missions assignées à l'institution scolaire, celle de l'éducation à la santé (ES) semble prendre une place significative à travers les programmes et les textes de cadrage publiés au cours des dix 
Tableau I. Évaluation du déroulement de la formation ESF par les adolescents $(n=21)$. Teenagers evaluation of the ESF $(n=21)$.

\begin{tabular}{|c|c|c|c|}
\hline No. & Propositions & Total en désaccord & Total en accord \\
\hline 3 & Le temps consacré aux explications et ateliers pratiques a été suffisant & $n=1(4,8 \%)$ & $n=20(95,3 \%)$ \\
\hline 1 & Le lieu où s'est déroulée la formation a été satisfaisant & $n=2(9,5 \%)$ & $n=19(90,5 \%)$ \\
\hline 2 & Le temps consacré à cette formation est suffisant & $n=3(14,3 \%)$ & $n=18(85,7 \%)$ \\
\hline 7 & Cette formation m’a donné envie de passer le brevet de secouriste & $n=4(19,1 \%)$ & $n=17(81 \%)$ \\
\hline 9 & Les ateliers pratiques sont applicables une fois de retour à domicile & $n=4(19,1 \%)$ & $n=17(81 \%)$ \\
\hline 5 & Les supports utilisés par les formateurs étaient adaptés & $n=5(23,9 \%)$ & $n=16(76,2 \%)$ \\
\hline 11 & Cette formation à la santé correspond à mes besoins et ceux de ma famille & $n=5(23,9 \%)$ & $n=16(76,2 \%)$ \\
\hline 13 & Cette formation me permettra de mieux parler de ma santé au quotidien dans ma famille & $n=5(23,9 \%)$ & $n=16(76,2 \%)$ \\
\hline 4 & Les échanges d'expériences entre les participants ont été constructifs & $n=6(28,6 \%)$ & $n=15(71,4 \%)$ \\
\hline 6 & J'ai appris des choses que je ne connaissais pas & $n=7(33,3 \%)$ & $n=14(66,7 \%)$ \\
\hline 15 & Globalement, cette formation a répondu à mes attentes & $n=7(33,3 \%)$ & $n=14(66,7 \%)$ \\
\hline 8 & Les formateurs m’ont donné la possibilité de m’exprimer sur mes expériences & $n=10(47,6 \%)$ & $n=11(52,4 \%)$ \\
\hline
\end{tabular}

Tableau II. Sentiment de compétence perçu suite à une ESF par les adolescents $(n=21)$. - Teenagers competences perceived after the ESF $(n=21)$.

\begin{tabular}{|c|c|}
\hline Je me sens capable de... & $n=21$ \\
\hline Mettre une personne en position latérale de sécurité (PLS) & $100 \%$ \\
\hline $\begin{array}{c}\text { Soigner une petite coupure } \\
\text { Soigner un mal de gorge } \\
\text { Soigner une toux } \\
\text { De mettre en place à domicile des mesures pour éviter une chute }\end{array}$ & $>90 \%$ \\
\hline $\begin{array}{c}\text { Réaliser un bandage, un pansement } \\
\text { Savoir quoi faire pour mon entourage en cas de canicule, de grand froid } \\
\text { Prendre en charge et calmer les pleurs d'un bébé } \\
\text { Comprendre le comportement d'une personne âgée } \\
\text { Soigner une piqûre d'insecte } \\
\text { Trouver les informations nécessaires à mes questions sur la santé } \\
\text { Savoir agir en cas de chute }\end{array}$ & $>80 \%$ \\
\hline $\begin{array}{c}\text { Surveiller et faire baisser la température d'un enfant } \\
\text { Réagir en cas de malaise d'une personne } \\
\text { Organiser une pharmacie de premiers soins à la maison } \\
\text { Commencer un massage cardiaque } \\
\text { Soulager des brûlures d'estomac } \\
\text { Soigner un rhume, une rhinite allergique } \\
\text { Donner des conseils de santé à ma famille et à mon entourage }\end{array}$ & $>70 \%$ \\
\hline $\begin{array}{c}\text { Gérer les médicaments de parents âgés } \\
\text { Soigner une (phlyctène) ampoule } \\
\text { Prendre en charge les coliques d'un bébé }\end{array}$ & $>60 \%$ \\
\hline $\begin{array}{c}\text { Orienter un copain qui a des problèmes d'addiction ou psychologiques } \\
\text { Calmer une douleur articulaire } \\
\text { Soigner une poussée de bouton de fièvre }\end{array}$ & $>50 \%$ \\
\hline Démarrer les premiers soins pour une foulure & $33 \%$ \\
\hline
\end{tabular}

Tableau III. Évaluation de la formation ESF trois mois plus tard par les adolescents $(n=21)$. Teenagers evaluation of the ESF three months later $(n=21)$.

\begin{tabular}{clcc}
\hline No. & Propositions & Total en désaccord & Total en accord \\
\hline 9 & Les apprentissages pratiques ont été applicables une fois de retour à domicile & $n=2(15,38 \%)$ & $n=11(84,61 \%)$ \\
\hline 12 & Cette formation m'a permis d'améliorer la prise en charge de la santé de ma famille & $n=5(38,46 \%)$ & $n=7(53,85 \%)$ \\
\hline 13 & Cette formation m'a permis de mieux aborder les sujets de santé au quotidien dans ma famille & $n=6(46,15 \%)$ & $n=7(53,85 \%)$ \\
\hline 15 & Globalement, cette formation a répondu à mes attentes & $n=5(38,46 \%)$ & $n=8(61,54 \%)$ \\
\hline
\end{tabular}


Tableau IV. Sentiment de compétence perçue, trois mois post-formation par les adolescents $(n=21)$. Teenagers competences perceived three months after the ESF training $(n=21)$.

\begin{tabular}{|c|c|c|}
\hline $\begin{array}{c}\text { Maux de santé } \\
\text { rencontrés }\end{array}$ & $\begin{array}{c}\text { Sentiment de pouvoir } \\
\text { agir }\end{array}$ & $\begin{array}{c}\text { Sentiment de ne pas } \\
\text { savoir agir }\end{array}$ \\
\hline Un rhume & Mettre en PLS & Un arrêt cardiaque \\
Une plaie, lors d'une & Les maux de ventre & Une fracture \\
chute & Les coupures & Une perte de \\
Une brûlure, en & Une brûlure & conscience \\
cuisinant & Une gastroentérite & \\
& Les petits maux de tête & \\
& Les vomissements & \\
& La toux & \\
& Trier les médicaments & \\
& de la pharmacie & \\
\hline
\end{tabular}

dernières années [24]. Néanmoins, ces textes semblent rester inopérants car ils ne se sont que peu ou pas accompagnés d'actions visant à impliquer réellement l'ensemble des acteurs de l'école.

Goigoux [25] affirme que les autorités de l'Éducation Nationale ont laissé les enseignants réaliser des «bricolages » didactiques et pédagogiques au lieu de les aider à constituer un véritable continuum entre des enseignements sur la santé relevant d'une approche scolaire traditionnelle et des pratiques davantage socio constructivistes centrées sur l'apprentissage des élèves à porter soins et secours dans une perspective citoyenne en santé.

Pour ces auteurs, il s'agit désormais d'apporter aux élèves des connaissances scientifiques sur les comportements à risque pour la santé dans une logique «prescriptive» et par ailleurs, de prendre en compte l'élève dans une approche globale afin de l'aider dans la construction de compétences en santé. Cependant, pour Lebaume [26], l'éducation pour la santé n'entre pas à l'école dans un cadre didactique clairement défini ; il explique qu'elle s'inscrit davantage dans une forme non scolaire de savoirs car elle ne dispose pas d'un programme disciplinaire clair en termes d'objectifs, de contenus et de tâches [27]. O'Neill relève trois difficultés pour la mise en place par l'institution scolaire de cette éducation [28] :

- la difficulté d'interagir entre le monde de l'éducation et celui de la santé, du fait que l'institution scolaire française est dotée de son propre service scolaire de santé ;

- le fait que les messages de promotion de la santé y sont encore très largement conçus comme une instruction, un apport de connaissances et non comme une appropriation de compétences permettant de devenir acteur de sa santé ;

- une formation réduite des personnels scolaires dans ce champ, qui aiderait au développement chez les élèves de compétences en santé.

Dans ce contexte, Pizon et al. [29] concluent que l'engagement des enseignants et des personnels scolaires relève souvent davantage d'une forme de militantisme et d'une conviction personnelle. Ils soulignent l'absence de programme de formation bien établi sur la santé citoyenne, ce qui pourrait créer un sentiment d'incertitude, voire d'incompétence chez le personnel enseignant [29]. Pour Berland [30], aujourd'hui encore, les jeunes de 12 à 18 ans sont exposés à des risques spécifiques mais ne font pour cela l'objet d'aucune attention systématique s'exprimant par une éducation à «des actions » en santé qui pourraient mettre à profit leurs contributions au quotidien.

Cette problématique a conduit en 2013 à l'une des principales dispositions de la Stratégie nationale de santé [31] portant sur la mise en place d'un «parcours éducatif en santé » à l'attention de la jeunesse devant être pour Lombrail [32] associée pour sa réussite avec la loi de refondation de l'école. Basée sur les recommandations du Conseil de l'Europe qui avait préconisé la mise en place de dispositifs d'apprentissages pour les familles, de l'éducation informelle, notamment pour les plus jeunes et sur le plan «Santé des jeunes» de 2008 [33,34], cette stratégie se donne pour ambition de permettre aux jeunes de :

« devenir acteur de leur santé en contribuant à la construction des valeurs morales et au réajustement des normes comportementales nécessaires à la sauvegarde de la santé».

Les auteurs de ce plan préconisent :

"plutôt que d'un saupoudrage d'informations, les collégiens et les lycéens ont besoin surtout d'un lieu de parole et d'échange identifié avec comme intervenants des professionnels formés (éducateurs, infirmières, psychologues, médecins, acteurs de la prévention... )»

Dès la fin des années 90, le LEPS EA 3412 en partenariat avec des urgentistes et des décideurs du Ministère de l'Éducation Nationale, avait proposé une approche éducative destinée à promouvoir la santé citoyenne des jeunes. Cette approche s'inscrivait déjà dans le cadre d'un «parcours éducatif en santé » tel qu'il est envisagé actuellement. Ce programme : «Apprendre à porter secours » [6] promeut l'apprentissage du secourisme de la maternelle au collège sur la base du volontariat au niveau des Académies et des départements. Il est désormais passé dans la loi.

Fort de cette expérience, le LEPS, a donc élaboré depuis 2006 un nouveau modèle d'éducation à la santé familiale [7-9] qui semble pouvoir répondre au besoin de l'éducation informelle en santé recommandé par le Conseil de l'Europe (2003) puisque cette éducation intéresse à présent les jeunes du collège au lycée [32]. Ce modèle est issu des travaux 
d'un partenariat original associant un laboratoire universitaire de recherche (LEPS EA 3412), un organisme du champ social (UNAF), un représentant du champ éducatif (MFR) et une caisse d'assurance sociale (MSA). Ces différents partenaires partagent la conscience d'une nécessaire émancipation des personnes et des familles à prendre en charge leur santé. Ils se sont alliés dans des échanges de construction ayant abouti à la mise sur pied de l'ESF. Cette dynamique de coopération a constitué un catalyseur indispensable à la réussite de l'expérience.

L'expérimentation de ce modèle à Conty a apporté des résultats encourageants, puisque trois mois après la formation la satisfaction des adolescents s'élevait à 61,54\% lorsqu'ils ont pu mettre en application au sein de leur famille ce qu'ils avaient appris et que 66,7\% de ceux qui ont participé à la formation ESF ont trouvé qu'elle avait répondu à leurs attentes.

Cette expérimentation répond aux recommandations du plan "Santé jeune » [33] en ayant formalisé deux jours dédiés à l'ESF sur le planning de cours d'une classe de lycéens et en proposant également un module de formation spécifique «Agir en tant que jeune », permettant aux adolescents de développer leur autonomie en santé tout en apportant une attention à celle des autres.

La pédagogie, qui a été mise en œuvre dans cette ESF, a sans doute constitué une rupture par rapport aux messages d'interdits trop souvent proposés par les programmes traditionnels de l'éducation à la santé en milieu scolaire [15] en y abordant «des actions » pour sa santé comme le préconise Berland [31]. De plus, des personnels formés en ESF : un médecin et un psychologue, extérieurs à la MFR sont intervenus pour collaborer avec un enseignant de mathématiques de l'institution pour animer les interventions éducatives. Ce partenariat a pu faciliter le transfert et le partage des connaissances entre formateurs. Cette interaction fructueuse entre le monde de la santé et celui du scolaire à un niveau éducatif [28] a permis de lever les deux autres difficultés évoquées précédemment : le manque de formation des enseignants et l'absence d'un programme de formation en santé préétabli [29]. Notre proposition d'intégrer l'ESF, aux institutions scolaires volontaires, permettrait de répondre à la circulaire de 2011 de l'Éducation nationale pour celles n'ayant pas développé une politique de "santé éducative structurée » avec un programme modélisé ; ce qui est encore majoritairement le cas sur le territoire [35].

Pizon et Berger expliquent que les établissements scolaires s'adaptent à l'évolution de leurs contextes et aux attentes de la société et qu'il s'agit à présent pour eux de développer une approche globale du «parcours éducatif en santé » en continu de la maternelle à l'université articulé avec les partenaires territoriaux [36]. Dans notre intervention, 69,87\% des adolescents ont été favorables à ce que l'ESF devienne un enseignement optionnel au Baccalauréat. Intégrer l'ESF dans un parcours éducatif en santé en milieu scolaire, permettrait également, selon nous, de répondre aux deux souhaits émis en 2012 par le CESE [37]. D'abord en soutenant et privilégiant la prévention par des pairs, en particulier chez les adolescents. En effet, les collégiens des classes de $3^{\mathrm{e}}$ ayant bénéficié d'une ESF pour- raient intervenir dans celles de leurs camarades de $6^{\mathrm{e}}$ et les lycéens des classes de terminales dans celles de seconde, par exemple Et enfin, le CESE insiste sur la nécessité de renforcer l'efficacité de la diffusion des messages élaborés par l'Inpes, qui ont été intégrés dans les interventions éducatives ESF.

\section{Conclusion}

Cette expérience d'ESF, s'inscrit clairement dans les perspectives portées par la nouvelle Stratégie nationale de santé et les politiques de santé publique en faveur de la prévention et de la promotion de la santé familiale et celle des jeunes en particulier. Il s'agit non seulement de faciliter le transfert et le partage des connaissances entre les professionnels de la santé et ceux de l'école mais également d'y associer les élèves adolescents. Ainsi, on pourrait envisager que l'ESF trouve sa place dans les nouveaux espaces temps des programmes scolaires D'autre recherches menées sur des groupes plus importants d'adolescents seront nécessaires pour montrer que l'ESF se démarque des interventions traditionnelles d'éducation en santé qui se focalisent pour la plupart sur des «mises en garde » ou celles plus récentes centrées sur les compétences psychosociales L'ESF place les adolescents en position d'acteurs en santé par l'apport immédiat de compétences visibles et applicables dans leurs contextes de vie familial et entre pairs

Remerciements. Les auteurs remercient les personnes impliquées dans cette expérimentation : le docteur Marie Van der Schueren-Etévé et Yoann Vasnier du PPEP. Louisette Régnier (Directrice), Pascal Haduck (Enseignant) et les lycéens de la MFR à Conty. Maria Grazia Albano et Pascal Cruchet pour leur aide à la traduction et à la révision du texte.

Conflits d'intérêts. L'auteur ne déclare aucun conflit d'intérêts concernant les données de cette étude.

\section{Références}

1. Durning P. Education familiale, acteurs, processus et enjeux. Paris : L'Harmattan ; 2006.

2. Cresson G. Le travail domestique de santé : analyse sociologique. Paris : L'Harmattan ; 1995.

3. Ivernois (d') JF, Gagnayre R. L'éducation au secours de la santé. La lettre de la SETE, 2006 ; 10, 1-2.

4. Ministère de la Santé et des Affaires Sociales. (2001). Plan national d'éducation pour la santé. Paris : France.

5. Ivernois (d') JF, Gagnayre R. Apprendre à éduquer le patient : apprendre pédagogique. $4^{\mathrm{e}}$ éd. Paris : Maloine ; 2011.

6. Ammirati C, Gagnayre R. Porter secours de la maternelle au collège : guide de l'enseignant. $2^{\mathrm{e}}$ éd. Paris : Maloine ; 2002.

7. Ivernois (d') JF, Gagnayre R, Rodary E, Brun N. Éducation des familles à Porter Soins et Secours: un nouveau concept dans le champ de l'éducation en santé. Education Thérapeutique du Patient 2010 ; 2(1):1-6.

8. Tessier S. Les éducations en santé. Paris : Maloine ; 2012. 
9. Riquet S. L'éducation à la santé familiale : modélisation et expérimentation de nouvelles formations à porter soins et secours. Thèse de doctorat és sciences en santé publique (sous la direction de d'Ivernois JF.). Université Paris 13, Sorbonne Paris Cité, 2015.

10. Mazué N. Education des familles et des adolescents à porter soins et secours. Etude de besoins sur un territoire de santé rural. Mémoire de Master 2 professionnel, Université Paris 13, Sorbonne Paris Cité, 2011.

11. Riquet $\mathrm{S}$. Besoins des familles vis-à-vis de l'éducation à porter soins et secours. Mémoire de Master 2 recherche, Université Paris 13, Sorbonne Paris Cité, 2010.

12. Hainaut (d') L. Programmes d'études et éducation permanente. Paris : UNESCO ; 1979.

13. Mucchielli R. Les méthodes actives, dans la pédagogie des adultes. $11^{\mathrm{e}}$ éd. Issy-les-Moulineaux : ESF éditeur ; 2008.

14. Meirieu P. L'école mode d'emploi, des méthodes actives à la pédagogie différenciée. 14éd. Paris : ESF éditeur ; 2014.

15. Roussille B. Soixante ans d'éducation pour la santé en France. La santé de l'homme $2002 ; 362: 14-20$.

16. Barlow M. Le travail de groupe des élèves. Paris : Armand Colin ; 1993.

17. Peeters L. Méthodes pour enseigner et apprendre en groupe. Bruxelles : De Boeck ; 2009.

18. Clark C. Brainstorming. How to Create Successful Ideas. Chatsworth, CA : Wilshire Book Company ; 1989.

19. Dupuis G, Gravel A, Jubinville C, Vachon D. L'exposé oral interactif. Québec français 1997; 107:67-70.

20. Peretti (de) A, Legrand JA, Boniface J. Techniques pour communiquer. Paris : Hachette ; 1994.

21. Baptiste A, Bélisle C, Péchenart JM, Vacheret C. Photolangage ${ }^{\circledR}$, une méthode pour communiquer en groupe par la photo. Paris : d'Organisation ; 1991.

22. Viollet P. Méthodes pédagogiques pour développer la compétence. Manuel pratique à l'usage des formateurs. Bruxelles : De Boeck ; 2011.

23. Bousquet V, Caserio-Schönemann C, \& les membres du comité de pilotage OSCOUR (2013). La surveillance des urgences par le réseau OSCOUR. Saint-Maurice : Institut de veille sanitaire.

24. Berger D, Pizon F, Bencharif L, Jourdan D. Education à la santé dans les écoles élémentaires... Représentations et pratiques enseignantes. Didaskalia 2009; 34:35-66.
25. Goigoux R. L'étude du travail de l'enseignant en didactique du français : le cas du maître de lecture. In: Mercier A (éd.). Balises pour la Didactique des Mathématiques. Grenoble : La pensée sauvage ; 2004.

26. Lebeaume J. Les manuels scolaires : des sources particulières pour l'étude curriculaire des sciences et des techniques à l'école. IOSTE Congress ; 2007, Critical analysis of sciences texbooks, Hammamet, Tunisie.

27. Lebeaume J. Perspectives curriculaires en éducation technologique. Mémoire d'habilitation à diriger des recherches, Université Paris Sud, 1999.

28. O'Neill M. Préface. In: Education à la santé. Quelle formation pour les enseignants ? Jourdan D. Saint-Denis : Inpes, Coll. Santé en action ; 2010.

29. Pizon F, Jourdan D, Simar C, Berger D. (2011). Les déterminants des pratiques d'éducation à la santé à l'école primaire : essai de catégorisation à partir du point de vue des enseignants. Travail et formation en éducation, $6:$ mis en ligne le 18 janvier. [Accès] : http://tfe.revues.org/1327 (consulté le 14 mars 2015)

30. Berland Y. Prise en charge de la santé de l'enfant. Synthèse des travaux de la commission, Paris : ONDPS ; 2013.

31. Ministère des Affaires Sociales et de la Santé. (2013). Stratégie nationale de santé, feuille de route. Paris, France.

32. Lombrail P. Orientation du projet de loi de santé : aller plus loin pour répondre aux défis structurels du système de santé. Santé Publique $2014 ;$ 4(26):475-480.

33. Comité des ministres aux Etats membres (2003). La promotion et la reconnaissance de l'éducation non formelle des jeunes. Recommandation No. 8. [Accès] : http://www.coe.int/t/dg4/ youth/Source/IG_Coop/Documents/CM_Rec_03_non_formal_ education_fr.pdf (consulté le 23 août 2014)

34. Ministère de la Santé de la Jeunesse et des Sports. (2008). Présentation du plan "Santé des jeunes". Paris : France.

35. Berger D, Bristol P, Morand J. Promotion de la santé des élèves dans les établissements scolaires. La santé en action : INPES 2014; 427(3):14-15.

36. Pizon F, Berger D. Promotion de la santé à l'école : de la théorie à la pratique. La santé en action : INPES 2014; 427(3):16-18.

37. Conseil Economique, Social et Environnemental. (2012). Les enjeux de la prévention en matière de santé. Avis présenté par Etienne JC. \& Corne C. Paris : France. 